Estimating two-point statistics from derivatives of a signal containing noise: Application to auto-correlation functions of turbulent Lagrangian tracks

N. Machicoane, P. D. Huck, and R. Volk

Citation: Review of Scientific Instruments 88, 065113 (2017); doi: 10.1063/1.4986467

View online: http://dx.doi.org/10.1063/1.4986467

View Table of Contents: http://aip.scitation.org/toc/rsi/88/6

Published by the American Institute of Physics

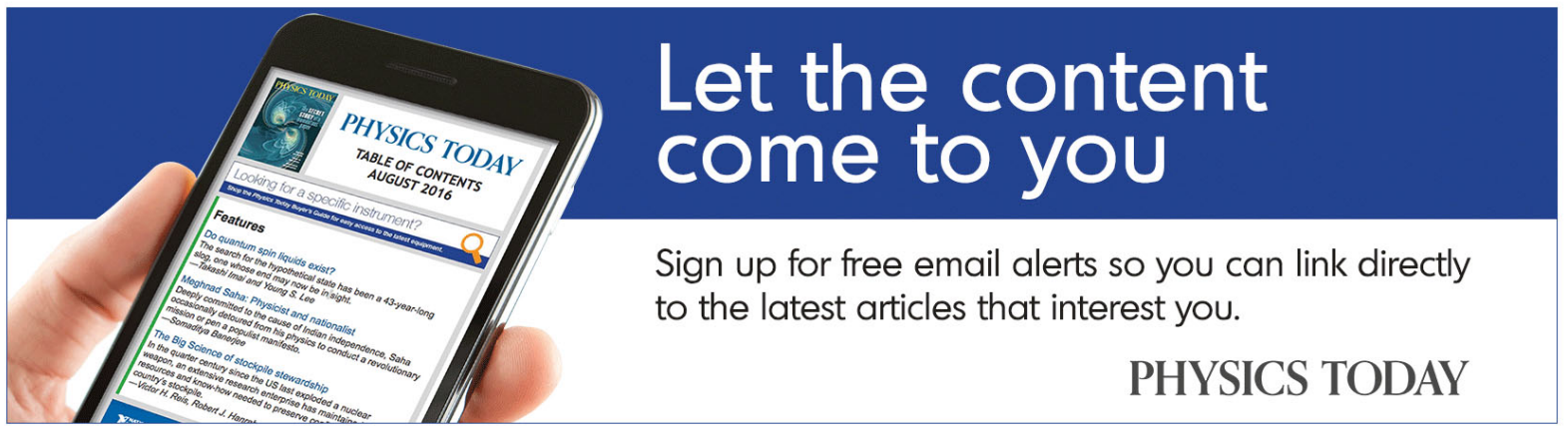




\title{
Estimating two-point statistics from derivatives of a signal containing noise: Application to auto-correlation functions of turbulent Lagrangian tracks
}

\author{
N. Machicoane, ${ }^{1, a)}$ P. D. Huck, ${ }^{2}$ and R. Volk ${ }^{2}$ \\ ${ }^{1}$ Department of Mechanical Engineering, University of Washington, Seattle, Washington 98195-2600, USA \\ ${ }^{2}$ Laboratoire de Physique, ENS de Lyon, UMR CNRS 5672, Université de Lyon, Lyon, France
}

(Received 24 February 2017; accepted 4 June 2017; published online 27 June 2017)

\begin{abstract}
This article describes a method for calculating moments and correlation functions of signal derivatives, which were rid of experimental noise without the use of filtering operations. The method is based on the computation of the ensemble-average of different time (or spatial) increments of the signal. The hypotheses are that the noise is white and not correlated with the signal; however, the method is also shown to work with colored noise. The method is first developed, considering white noise, and benchmarked with synthetic trajectories containing noise with variable signal-to-noise ratios. It is then tested on experimental trajectories in the context of Lagrangian tracking of particles in turbulent flows, either containing a short-correlated noise or a colored noise. Published by AIP Publishing. [http://dx.doi.org/10.1063/1.4986467]
\end{abstract}

\section{INTRODUCTION}

Any experimental measurement is subject to noise. While the level of noise can generally be reduced by finely tuning the experimental setup, the noise level in the signal's derivatives inevitably increases with the number of derivations, making estimation of their moments and correlations difficult. A common solution is to filter the signal prior to differentiation, as was already done for digital circuit differentiator, ${ }^{1}$ but great care has to be taken concerning the choice of the filter width and type in order to minimize the signal alteration. ${ }^{2}$ By trying to remove the noise, the experimentalist incorporates a subjective bias into the data, which is unfortunately unavoidable in certain analyses. However, when concerned by moments and correlation functions of signal derivatives, we show in this article that it is possible to directly remove the noise and access unbiased quantities as long as the noise is not correlated with the measured signal.

Classical sources of noise in a measurement can be external perturbations, such as variations of the lighting when using a digital camera or sensor inconsistency. Such noises can often be described as a white noise, uncorrelated with the signal. By assuming the latter, we show that the proposed method works in this simple framework and also in the case of colored noise. We have conducted this approach in the context of Lagrangian particle tracks in turbulent flows which are non-stationary temporal signals, but the method is valid for any signal, depending either on space or time. It is based on the computation of the ensemble-average of different time (or spatial) increments of the signal and is inspired by previous works. ${ }^{3,4}$ Velocimetry measurements based on the analysis of the motion of particles have become the most commonly used metrology technique in contemporary fluid mechanics research. ${ }^{5}$ For instance, particle tracking velocimetry consists of recording particle positions

\footnotetext{
a) Electronic mail: nathanael.machicoane@ens-lyon.org
}

in successive images using digital cameras, ${ }^{6}$ while Extended Laser Doppler Velocimetry (ELDV) directly yields particle velocity trajectories using a photomultiplier and two crossing laser beams. ${ }^{7}$ In the first case, the proper hardware (camera spatial and time resolution, uniform lighting) and software (camera calibration model, ${ }^{8}$ trajectory construction ${ }^{9,10}$ ) considerations ensure that the noise is short-correlated and uncorrelated with the signal. For the second case, however, the particle velocity tracks are extracted from Doppler signals with a recursive estimation using a Kalman filter, ${ }^{7,11}$ so that the noise is colored. In both cases, the signal is usually time-filtered prior to differentiation or a filtering-differentiating kernel is used, ${ }^{12,13}$ which increases the signal-to-noise ratio at the cost of a signal alteration. The goal of the proposed variable time step method is to avoid such filtering operations. Before testing the method for those two experimental cases, we have designed a benchmark case where the noise is added synthetically to position trajectories of tracer particles from a direct numerical simulation of turbulence. ${ }^{14}$ This allows for the direct comparison of the quantities estimated from the proposed method to the one obtained from the signal prior to noise addition. As the three test cases deal with a similar physical phenomenon, they yield statistics (in particular correlation functions) that behave similarly, constituting a common framework in which the proposed method is tested.

This article is organized as follows. Section II presents the method and its implementation, which is assessed by the numerical benchmark in Sec. III. The method is then applied to both experimental measurements and the results are compared to what is obtained by filtering considerations in Sec. IV, which is followed by a discussion (Sec. V).

\section{SIGNAL PROCESSING}

\section{A. Second order moments}

The method presented below seeks to obtain unbiased one and two-point statistics of the derivatives of an 
experimental signal without introducing any filtering method. It is valid for any measured signal whose typical correlation scale is much larger than the noise correlation scale. While one aims at obtaining the real signal $\hat{x}$, the presence of noise $b$ implies that one measures $x(t)=\hat{x}+b$. For simplicity, we consider here the case of a temporal signal $x(t)$ that is centered, i.e. $\langle x\rangle=0$, which can be recovered by considering $x(t)-\langle x\rangle$, where $\langle$.$\rangle is an ensemble average.$

The method is based on the temporal increment $d x$ of the signal $x$ over a time $d t$, which we express as $d x=x(t+d t)-x(t)=d \hat{x}+d b$. Assuming that the increments of position and noise are uncorrelated, the variance of the position increment is $\left\langle(d x)^{2}\right\rangle=\left\langle(d \hat{x})^{2}\right\rangle+\left\langle(d b)^{2}\right\rangle$. Introducing the velocity $\hat{v}$ and acceleration $\hat{a}$ through a second order Taylor expansion $\hat{x}(t+d t)=\hat{x}(t)+\hat{v} d t+\hat{a} d t^{2} / 2+o\left(d t^{2}\right)$, one obtains

$$
\left\langle(d x)^{2}\right\rangle=\left\langle(d b)^{2}\right\rangle+\left\langle\hat{v}^{2}\right\rangle d t^{2}+\langle\hat{a} . \hat{v}\rangle d t^{3}+o\left(d t^{3}\right),
$$

where $\left\langle(d b)^{2}\right\rangle=2\left\langle b^{2}\right\rangle$ in the case of a white noise. In Eq. (1), $\left\langle(d x)^{2}\right\rangle$ is a function of $d t$ so that one can recover the value of the velocity variance $\left\langle\hat{v}^{2}\right\rangle$ by calculating time increments of $\left\langle(d x)^{2}\right\rangle(d t)$ over different values of $d t$ followed by a simple polynomial fit in the form of Eq. (1). If the noise is colored, $\left\langle(d b)^{2}\right\rangle=2\left\langle b^{2}\right\rangle-2\langle b(t) b(t+d t)\rangle$. The method hence requires the noise to be correlated only on short times compared to the signal correlation time, so that only the lowest values of $\left\langle(d x)^{2}\right\rangle(d t)$ are biased by $\langle b(t) b(t+d t)\rangle$ and a fit still successfully allows for the evaluation of the root mean square (rms) velocity, $\hat{v}^{\prime}=\sqrt{\left\langle\hat{v}^{2}\right\rangle}$. For an experimentally measured signal $x$, equally spaced at an acquisition $\operatorname{rate} f_{s}$, the minimal value of $d t$ is $1 / f_{s}$; we can then obtain the values of $d x$ for different values of $d t=n / f_{s}$. For this method, we need a value of the acquisition rate $f_{s}$ that is purposefully higher than usual, in order to be able to access derivatives of the signal without aliasing error.

We can extend the previous calculation to higher order derivative statistics by considering higher order increments. The second order increment $d^{2} x=x(t+d t)+x(t-d t)-2 x(t)$, which is related to the acceleration variance $\left\langle\hat{a}^{2}\right\rangle$ here, yields, for instance,

$$
\left\langle\left(d^{2} x\right)^{2}\right\rangle=\left\langle\left(d^{2} b\right)^{2}\right\rangle+\left\langle\hat{a}^{2}\right\rangle d t^{4}+\frac{1}{6}\left\langle\hat{a} \cdot \frac{d^{2} \hat{a}}{d t^{2}}\right\rangle d t^{6}+o\left(d t^{6}\right),
$$

where $\left\langle d^{2} b^{2}\right\rangle=6\left\langle b^{2}\right\rangle$ in the case of a white noise, but otherwise introduces additional noise correlation terms which are functions of $d t$.

\section{B. Auto-correlation functions}

The approach developed above is not restricted to one time statistics of the signal derivatives but can be generalized to estimate the first and second order derivative auto-correlation functions of the noiseless signal $C_{\hat{v} \hat{v}}=\langle\hat{v}(t) \hat{v}(t+\tau)\rangle$ and $C_{\hat{a} \hat{a}}=\langle\hat{a}(t) \hat{a}(t+\tau)\rangle$. This is done by considering the correlations of first and second order increments $\langle d x(t) \cdot d x(t+\tau)\rangle$ and $\left\langle d^{2} x(t) \cdot d^{2} x(t+\tau)\right\rangle$, which are functions of $d t$ and $\tau$. As was done in Sec. II B for the variance, noiseless correlation functions are estimated, for each time-lag $\tau$, from a polynomial fit of the signal time increment $d t$ with the respective expressions,

$$
\left\{\begin{array}{c}
C_{d x d x}(\tau, d t)=C_{\hat{v} \hat{v}}(\tau) d t^{2}+\frac{1}{2}\left(C_{\hat{v} \hat{a}}(\tau)+C_{\hat{a} \hat{v}}(\tau)\right) d t^{3}+C_{d b d b}(\tau, d t)+o\left(d t^{3}\right) \\
C_{d^{2} x d^{2} x}(\tau, d t)=C_{\hat{a} \hat{a}}(\tau) d t^{4}+\frac{1}{12}\left(C_{\hat{a}\left(d^{2} \hat{a} / d t^{2}\right)}(\tau)+C_{\left(d^{2} \hat{a} / d t^{2}\right) \hat{a}}(\tau)\right) d t^{6}+C_{d^{2} b d^{2} b}(\tau, d t)+o\left(d t^{6}\right),
\end{array}\right.
$$

where $C_{f g}=\langle f(t) g(t+\tau)\rangle$ is a cross correlation function. It can be noted that the case of the rms values corresponds to $\tau=0$, and we remind the reader that $\left\langle(d x)^{2}\right\rangle$ and $\left\langle\left(d^{2} x\right)^{2}\right\rangle$ are functions of $d t$. In the previous expressions and in the case of a white noise, we can express the auto-correlation functions of the noise as first and second order increments. The signal being sampled at a frequency $f_{s}$, one has $d t=n / f_{s}$ and $\tau=m / f_{s}$. The correlation functions of the digitized noise increments are written as

$$
\left\{\begin{array}{c}
C_{d b d b}\left(\tau=\frac{m}{f_{s}}, d t=\frac{n}{f_{s}}\right)=\left\langle b^{2}\right\rangle\left(2 \delta_{m, 0}-\delta_{m, n}\right), \\
C_{d^{2} b d^{2} b}\left(\tau=\frac{m}{f_{s}}, d t=\frac{n}{f_{s}}\right)=\left\langle b^{2}\right\rangle\left(6 \delta_{m, 0}-4 \delta_{m, n}+\delta_{m, 2 n}\right),
\end{array}\right.
$$

where $\delta_{m, n}$ is the Kronecker symbol. For both derivatives, the white noise magnitude in the first-order derivative autocorrelation functions is the highest for $\tau=0$ and is an additive term. The noise then yields a negative term for $m=n$. In the case of second-order derivatives (for acceleration in the case of Lagrangian tracks), the noise magnitude is larger and also contributes to a third time point of the function $(m=2 n)$ with a positive term of smaller amplitude. Considering white noise terms up to $d t^{6}$, all other values of $\tau$ will directly yield the function without noise.

\section{NUMERICAL TEST}

The numerical test uses tracer particles' trajectories in homogeneous isotropic turbulence from Refs. 15 and 16. The particles are tracked in a periodic box of dimension $L=2 \pi$, with $512^{3}$ grid points, in a turbulent flow at a Taylor-based Reynolds number $R e_{\lambda}=180$. The trajectories $\left(x_{p}(t)\right)_{p \in[1, N]}$ are multi-scale temporal signals with a smallest time scale $\tau_{\eta}$ and an integral time scale $T \simeq \tau_{\eta} \sqrt{R e_{\lambda}}$. In the present case, $\tau_{\eta}=0.047 \mathrm{~s}$ and $T=0.59 \mathrm{~s}$. Here, we use $N=2000$ trajectories of duration $170 \tau_{\eta} \gg T$ which contain acceleration signals measured at the particle positions at a frequency $f_{s}^{0}=11.75 / \tau_{\eta}$. These acceleration signals are therefore not obtained by derivation of particle positions and can be considered to be noiseless. For the purpose of the present test, a 

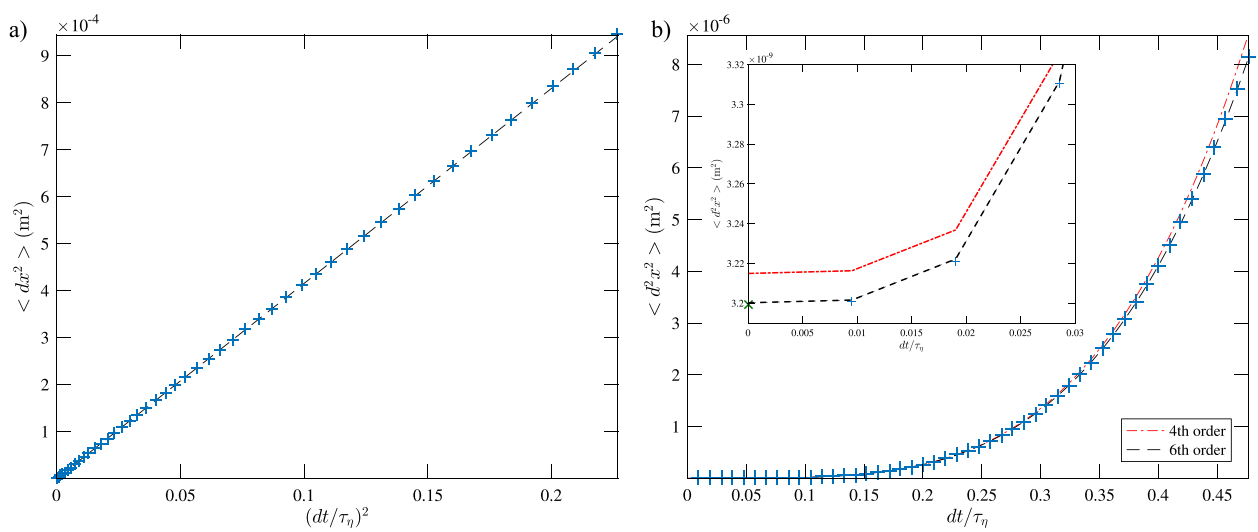

FIG. 1. (a) Evolution of $\left\langle(d x)^{2}\right\rangle$ with $\left(d t / \tau_{\eta}\right)^{2}$. The dashed line is a linear fit over the range $0<d t / \tau_{\eta} \leq 0.07$. (b) Evolution of $\left\langle\left(d^{2} x\right)^{2}\right\rangle$ with $d t / \tau_{\eta}$. The dashed-dotted and dashed lines are, respectively, fourth and sixth order fits $\left(\alpha+\beta d t^{4}\right.$, respectively, $\left.\alpha+\beta d t^{4}+\gamma d t^{6}\right)$ over the range $0<d t / \tau_{\eta} \leq 0.14$. The inset is a zoom on low values of $d t / \tau_{\eta}$; the green cross $(\times)$ at $d t=0$ in the inset corresponds to $6\left\langle\hat{b}^{2}\right\rangle$. higher sampling frequency is needed and we choose to interpolate acceleration signals so that the noiseless acceleration trajectories, $\left(\hat{a}_{p}(t)\right)_{p \in[1, N]}$, have a sampling frequency $f_{s}=10 f_{s}^{0}$. We then integrated them twice to obtain the noiseless position trajectories $\left(\hat{x}_{p}(t)\right)_{p \in[1, N]}$ to which a Gaussian random noise, $\hat{b}$, is added at each time step.

The method is tested on these noisy position trajectories and the result can then be compared to quantities computed on the originating signal of (interpolated) acceleration trajectories. To quantify the weight of the noise on the raw acceleration signal, we compute the noise-tosignal ratio as $\left(\hat{b}^{\prime} f_{s}^{2}\right) / \hat{a}^{\prime}=19.7$, where $\hat{b}^{\prime}=\sqrt{\left\langle\hat{b}^{2}\right\rangle}=23 \mu \mathrm{m}$ and $\hat{a}^{\prime}=\sqrt{\left\langle\hat{a}^{2}\right\rangle}=7.3 \mathrm{~m} \mathrm{~s}^{-2}$ are, respectively, the true rms value of the noise and acceleration, emphasizing the need of a method to eliminate this noise. While this value corresponds to a poor signal-to-noise ratio, it is of the order of what can be found in particles tracking experiments (see Sec. IV A).

\section{A. Moments and correlations}

Figure 1 shows the evolution of $\left\langle(d x)^{2}\right\rangle$ and $\left\langle\left(d^{2} x\right)^{2}\right\rangle$ with $d t$, where $\langle\cdot\rangle$ averages both over time (within a trajectory) and over the number of trajectories. As the velocity is only a first-order derivative, a low level of noise is observed $\left[\left(\hat{b}^{\prime} f_{s}\right) / \hat{v}^{\prime}=0.05\right]$ and a linear function of $d t^{2}$, without considering any higher order term, fits $\left\langle(d x)^{2}\right\rangle$ almost perfectly. For the acceleration, a sixth-order fit $\left[\alpha+\beta d t^{4}+\gamma d t^{6}\right.$, following Eq. (2)] is much better than considering a fourth-order regression, as is underlined in the inset of Fig. 1(b). This is expected because turbulent trajectories are known to exhibit two time scales: one characteristic of velocity changes and a much shorter one associated with acceleration (note that this is not specific of turbulence but should hold for any multi-scale signals). The range $d t / \tau_{\eta}$ (i.e., the number of points used in the regression) considered by those fits is chosen to maximize the fits agreement with the data and corresponds to the values of the absciss indicated in the caption of Fig. 1. The influence of this choice, while found not to be critical, is discussed in Subsection III B. The fact that the behavior of $\left\langle(d x)^{2}\right\rangle$ and $\left\langle\left(d^{2} x\right)^{2}\right\rangle$ follows closely the one given in Eqs. (1) and (2) is expected as this numerical test respects the hypotheses of the method (considering a white noise uncorrelated with the signal).

By fitting $\langle d x(t) \cdot d x(t+\tau)\rangle$ and $\left\langle d^{2} x(t) . d^{2} x(t+\tau)\right\rangle$ on each instant $\tau$ considered, as is done in Fig. 1 for $\tau=0$, we estimate the correlation functions, which are compared in Fig. 2 with their true counterparts (computed directly with noisefree velocity and acceleration signals). We obtain an excellent agreement in this benchmark case, where only a slight difference (respectively, $0.01 \%$ and $0.2 \%$ of maximal relative error) is observed for the auto-correlation functions of the first and second order derivatives.

\section{B. Robustness of the method}

This benchmark case allows for a quantitative comparison between what is estimated by the method and the real quantities. In order to do so, we define the acceleration time scale of the noiseless signal, $\tau_{\hat{a}}$, as the integral of the positive part of the acceleration correlation function normalized by the
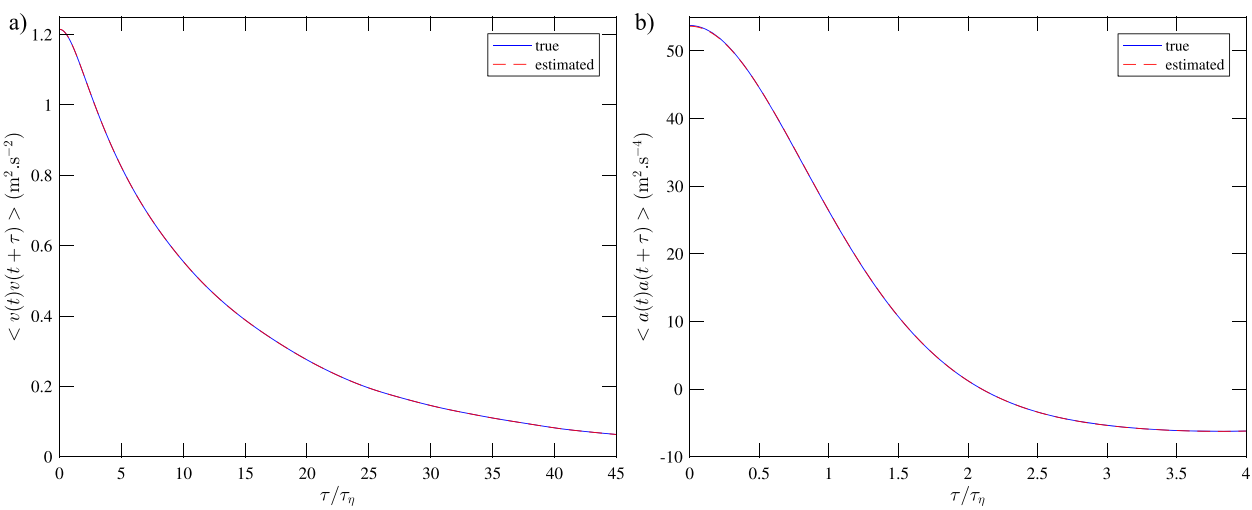

FIG. 2. Velocity (a) and acceleration (b) auto-correlation functions from the numerical data prior to noise addition (continuous line) and estimated from the proposed method on the noisy data (dashed line). The noise-to-signal ratio is $\hat{b}^{\prime} f_{s}^{2} / \hat{a}^{\prime}=19.7$. The fit ranges used to obtain the functions are the same than that used in Fig. 1. 
TABLE I. Relatives errors on the estimation of the variance of the acceleration and noise and on the estimation of the acceleration time scale for different (sixth order) fit ranges and a noise-to-signal ratio $\hat{b}^{\prime} f_{s}^{2} / \hat{a}^{\prime}=19.7 .\left\langle\hat{a}^{2}\right\rangle, \tau_{\hat{a}}$, and $\left\langle\hat{b}^{2}\right\rangle$ are the true quantities, and $\left\langle a^{2}\right\rangle, \tau_{a}$, and $\left\langle b^{2}\right\rangle$ are the quantities estimated with the method.

\begin{tabular}{lcrrrc}
\hline \hline \multicolumn{2}{c}{ Fit ranges } & & \multicolumn{3}{c}{ Relative errors } \\
\cline { 1 - 3 } \cline { 5 - 6 } No. of points & $(d t)_{\max } / \tau_{\eta}$ & & $\frac{\left\langle a^{2}\right\rangle-\left\langle\hat{a}^{2}\right\rangle}{\left\langle\hat{a}^{2}\right\rangle}$ & $\frac{\left\langle b^{2}\right\rangle-\left\langle\hat{b}^{2}\right\rangle}{\left\langle\hat{b}^{2}\right\rangle}$ & $\frac{\tau_{a}-\tau_{\hat{a}}}{\tau_{\hat{a}}}$ \\
\hline 7 & 0.07 & & $4.2 \times 10^{-3}$ & $6.8 \times 10^{-5}$ & 0.94 \\
15 & 0.14 & & $3.4 \times 10^{-5}$ & $2.3 \times 10^{-4}$ & $2.8 \times 10^{-5}$ \\
30 & 0.28 & & $4.8 \times 10^{-4}$ & $2.3 \times 10^{-3}$ & $4.9 \times 10^{-4}$ \\
50 & 0.47 & & $3 \times 10^{-3}$ & $9.6 \times 10^{-2}$ & $3.1 \times 10^{-3}$ \\
\hline \hline
\end{tabular}

acceleration variance. Table I presents the relative errors on the estimation of the acceleration, noise variance, and acceleration time scale evolution with the number of points used for the fits. While it is clear that an optimum value exists (around 15 in this case), one can appreciate that even using only the bare minimum of points or exaggerating, this number still yields very low errors (less than $0.4 \%$ for the acceleration variance). It means that the only criterion to choose this parameter should be the agreement between the fit and the data points, as long as the range considered remains physically relevant [in this example, $(d t)_{\max } / \tau_{\eta}$ should not be too close to 1 , so that $\left\langle\left(d^{2} x\right)^{2}\right\rangle$ is still relevant to an acceleration]. Note that the high error of $\tau_{a}$ when considering only 7 points is linked to the fact that the noise for $\tau=d t$ and $\tau=2 d t$ still strongly biases the acceleration correlation function integral. The fact that the noise variance error is a decreasing function of the point number is easily understood considering that $\left\langle\left(d^{2} x\right)^{2}\right\rangle$ is a polynomially growing function of $d t$; the more the points considered, the lesser is the weight given to the first points. This can be observed in the inset of Fig. 1(b) where the fit, by overestimation over the first few points because of their lesser weight, overestimates slightly the value of $\left\langle b^{2}\right\rangle$.

Lastly, we consider the impact of the noise magnitude, estimated as $\left(\hat{b}^{\prime} f_{s}^{2}\right) / \hat{a}^{\prime}$, on the accuracy of the method. Results are given in Table II. A first impact of this parameter is the need of more fit points to correctly estimate the acceleration time scale $\tau_{a}$. To obtain a relative error on $\tau_{a}$ below $1 \%$, we need to consider, respectively, at least $9,12,14$, and 16 points for the noise magnitudes shown in Table II. Note that this is not the

TABLE II. Relatives errors on the estimation of the variance of the acceleration and noise and on the estimation of the acceleration time scale for different noise magnitudes, with a fixed fit range of $(d t)_{\max } / \tau_{\eta}=0.15$ (16 fit points). $\left\langle\hat{a}^{2}\right\rangle, \tau_{\hat{a}}$, and $\left\langle\hat{b}^{2}\right\rangle$ are the true quantities, and $\left\langle a^{2}\right\rangle, \tau_{a}$, and $\left\langle b^{2}\right\rangle$ are the quantities estimated with the method.

\begin{tabular}{lccc}
\hline \hline & \multicolumn{3}{c}{ Relative errors } \\
\cline { 2 - 4 }$\hat{b}^{\prime} f_{s}^{2} / \hat{a}^{\prime}$ & $\frac{\left\langle a^{2}\right\rangle-\left\langle\hat{a}^{2}\right\rangle}{\left\langle\hat{a}^{2}\right\rangle}$ & $\frac{\left\langle b^{2}\right\rangle-\left\langle\hat{b}^{2}\right\rangle}{\left\langle\hat{b}^{2}\right\rangle}$ & $\frac{\tau_{a}-\tau_{\hat{a}}}{\tau_{\hat{a}}}$ \\
\hline 19.7 & $2.5 \times 10^{-5}$ & $2.3 \times 10^{-4}$ & $4 \times 10^{-6}$ \\
39.4 & $2.5 \times 10^{-4}$ & $2.6 \times 10^{-4}$ & $4.1 \times 10^{-6}$ \\
59.1 & $3.5 \times 10^{-4}$ & $4.9 \times 10^{-4}$ & $1 \times 10^{-5}$ \\
78.7 & $1.6 \times 10^{-4}$ & $1.3 \times 10^{-4}$ & $1 \times 10^{-5}$ \\
\hline \hline
\end{tabular}

case for the error on the acceleration (or noise) variance that stays below $0.4 \%$ whatever the noise magnitude even when considering only 10 fit points. Hence, we have used 16 points to compute the relative errors of this table. One can observe that even by multiplying the noise by about 4 compared to what has been considered in Subsection III A, the relative errors barely change. This shows that as long as the noise is white and uncorrelated with the signal, the proposed method should work, irrespective of the noise level. We emphasize that, with such low signal-to-noise ratios, it would probably be impossible to directly measure the statistics estimated here without a denoising strategy.

\section{EXPERIMENTAL TEST}

This section is devoted to the test of the method in two experimental configurations, where the noise is a priori either white (or correlated on much shorter times than the signal) or colored. Both cases consider Lagrangian tracks of particles in a turbulent von Kármán flow produced by two counter-rotating discs in a vessel filled with water.

\section{A. Shortly correlated noise}

This first case uses the raw position trajectories of material particles from Ref. 17. The method has been tested successfully for different particle diameters (from 6 to $24 \mathrm{~mm}$ ), Reynolds numbers $\left(350<R e_{\lambda}<520\right)$, and two density ratios (0.9 and 1.14), as well as for isodense particles from Ref. 18. We will focus only on the case of particles $6 \mathrm{~mm}$ in diameter and of density ratio 1.14 at a Reynolds number $R e_{\lambda}=520$ in the following. The position trajectories are obtained by stereomatching of successive image pairs obtained thanks to two cameras and global lighting. The particles appear as large, bright discs on an uniform dark background which yields subpixel noise for the trajectories (the apparent particle diameter is about 20 pixels) and is not correlated with the particle position as the background is uniform (nor with its velocity as the exposure time is short enough to fix the particles on the images). In practical situations, the presence of sub-pixel displacements can lead to a short-time correlation of the noise, typically on a few frames.

Figure 3 shows the evolution of $\left\langle(d x)^{2}\right\rangle$ and $\left\langle\left(d^{2} x\right)^{2}\right\rangle$ with $d t$. As with the numerical test, a simple linear function of $d t^{2}$ is enough for $\left\langle(d x)^{2}\right\rangle$ and a sixth-order one suits better $\left\langle\left(d^{2} x\right)^{2}\right\rangle$. The first points of $\left\langle\left(d^{2} x\right)^{2}\right\rangle$ do not follow Eq. (2), which may be due to the fact that we are not dealing with a purely white noise as will be shown in Fig. 4(b). Using the estimated values of the rms acceleration, $a^{\prime}$, and $\left\langle\left(d^{2} b\right)^{2}\right\rangle$, we can define a noise-to-signal ratio $b^{\prime} f_{s}^{2} / a^{\prime}=11.9$ where we have defined $b^{\prime}=\sqrt{\left\langle\left(d^{2} b\right)^{2}\right\rangle / 6}$ by analogy with the white noise case. When considering the noise weight on the velocity signals, we of course find a much smaller magnitude $b^{\prime} f_{s} / v^{\prime}=0.14$ as it is only a first order derivative ( $v^{\prime}$ being the rms of the velocity estimated with the method).

Figure 4 shows the auto-correlation function of both the velocity and acceleration estimated with the proposed method, 

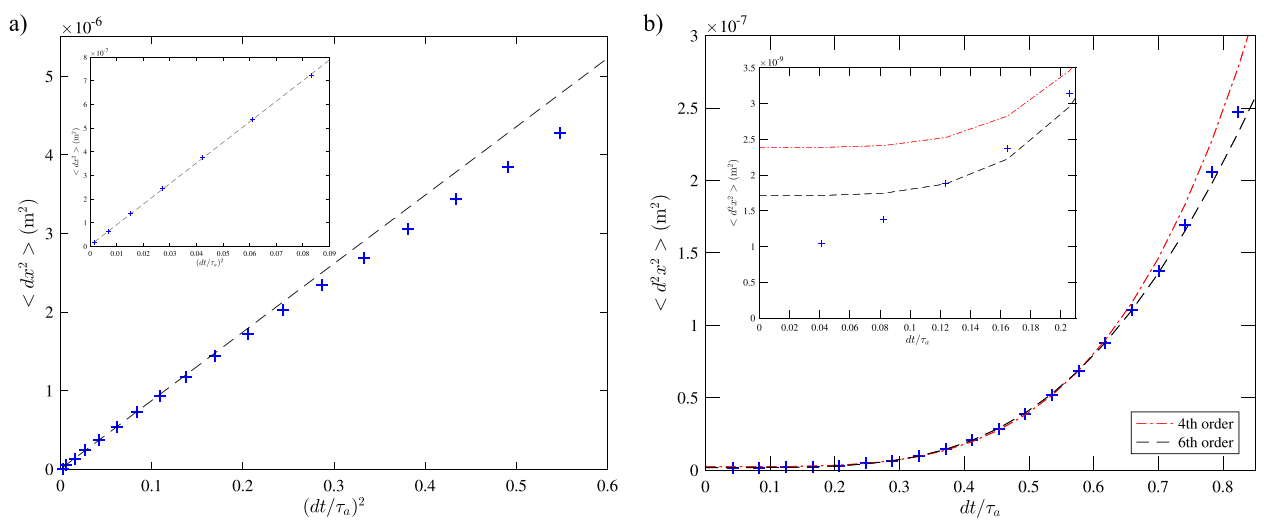

FIG. 3. (a) Evolution of $\left\langle(d x)^{2}\right\rangle$ with $\left(d t / \tau_{a}\right)^{2}$, where $\tau_{a}=8.1 \mathrm{~ms}$ is the particle acceleration time scale (integral of the positive part of the particle acceleration auto-correlation function). The dashed line is a linear fit over the range $0<d t / \tau_{a} \leq 0.25$. (b) Evolution of $\left\langle\left(d^{2} x\right)^{2}\right\rangle$ with $d t / \tau_{a}$. The dashed-dotted and dashed lines are, respectively, fourth and sixth order fits $\left[\alpha+\beta\left(d t / \tau_{a}\right)^{4}\right.$, respectively, $\left.\alpha+\beta\left(d t / \tau_{a}\right)^{4}+\gamma\left(d t / \tau_{a}\right)^{6}\right]$ over the range $0<d t / \tau_{a} \leq 0.62$. The insets are zooms on low values of $d t / \tau_{a}$.

compared to the raw functions. With the low level of noise in this configuration, the velocity is almost unbiased and both functions are indistinguishable except for the first points of the raw function that are offset by the noise. Concerning the second-order derivative quantity, it can be observed from Fig. 4(b) that the raw acceleration auto-correlation function is not biased only on the three first points (see inset). This is because the noise is not white but has a short correlation time compared to the signal. Combined with the finite duration of the trajectories, the raw correlation function is noisy over the whole range of time-lags $\tau$. This curve is plotted together with the one estimated with the method, fitting the coefficient up to $d t=5 \mathrm{~ms}$, which corresponds to $30 \%$ correlation loss in acceleration signals [same range as in Fig. 3(b), but the precise choice is not critical]. Although the signal-to-noise ratio is poor, the estimated correlation function seems to be following the median line between the peaks caused by noise and crosses zero at the location that seemed to be indicated by the raw function. It is also close to the auto-correlation function from Ref. 17, estimated by filtering the data with a Gaussian kernel $K=A_{w} \exp \left(-t^{2} / 2 w^{2}\right.$ ) (with $w=12$ points and a compact support of width $2 w$ ) and $A_{w}$ is a normalization factor. It should be stressed that the value $w=12$ was chosen arbitrarily as a compromise between suppressing oscillations at small lags without altering the shape of the function too much at larger lags.

With the new method, we compute an acceleration time scale $\tau_{a}=8.1 \mathrm{~ms}$ and an acceleration magnitude $a^{\prime}=12.4 \mathrm{~m} \mathrm{~s}^{-2}$, which is close to the values $\tau_{a}=8.8 \mathrm{~ms}$ and $a^{\prime}=12.9 \mathrm{~m} \mathrm{~s}^{-2}$ found for the filtered data. ${ }^{17}$ However, in the latter case, the value of $a^{\prime}$ depends strongly of the choice of the filter width $w$, so that one usually estimates $a^{\prime}$ by computing it for different filter widths which can then allow to extrapolate a best estimate value (as introduced in Ref. 13).

\section{B. Colored noise}

The second case considers raw velocity trajectories from Refs. 7 and 19 obtained by Extended Laser Doppler Velocimetry (ELDV) measurements of tracer particles. The velocity is directly obtained through a frequency demodulation by use of an approximate maximum likelihood method coupled to a Kalman filter. ${ }^{11}$ Although the signal is sampled at very high frequency $1 \mathrm{MHz}$ (the carrying frequency is $100 \mathrm{kHz}$ ), the instantaneous frequency is estimated over a short window (here $30 \mu \mathrm{s}$ ) and is influenced by its estimation at the previous time step. Even assuming that the noise, noted $b_{v}$, is initially white, it is low-pass filtered and becomes colored in the process. Applying the Taylor expansion to the present case $\hat{v}(t+d t)=\hat{v}(t)+\hat{a}(t) d t+\frac{1}{2} \frac{d \hat{a}}{d t} d t^{2}+o\left(d t^{2}\right)$, the correlation function of velocity increment is written as

$$
\begin{aligned}
\langle d \hat{v}(t) d \hat{v}(t+\tau)\rangle(\tau, d t)= & \left\langle d b_{v}(t) d b_{v}(t+\tau)\right\rangle+C_{\hat{a} \hat{a}}(\tau) d t^{2} \\
& +\frac{1}{2}\left(C_{\hat{a}(d \hat{a} / d t)}(\tau)+C_{(d \hat{a} / d t \hat{a}}(\tau)\right) d t^{3} \\
& +o\left(d t^{3}\right) .
\end{aligned}
$$

The acceleration is directly obtained from the first order derivative of the velocity here, so that $C_{\hat{a} \hat{a}}(\tau)$ is estimated using a fit of the form $\alpha_{v}+\beta_{v} d t^{2}+\gamma_{v} d t^{3}$ for each time-lag $\tau$.
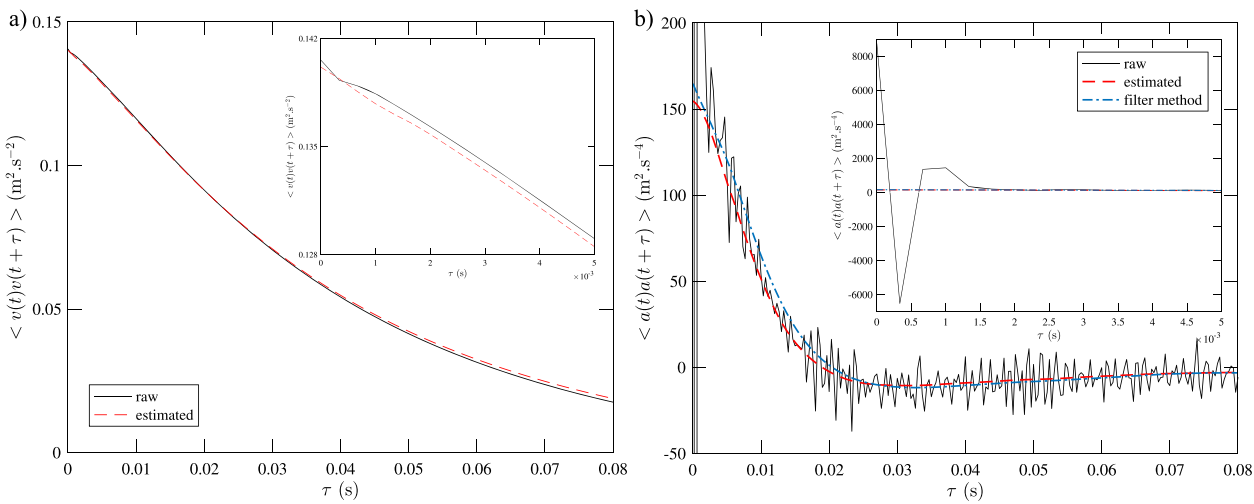

FIG. 4. Auto-correlation functions of the velocity (a) or acceleration (b) estimated from the proposed method (dashed line) and directly computed by differentiating the position signal obtained by PTV (continuous line). The insets are zooms on the low values of $\tau$. The fit ranges used to obtain the functions are the same than used in Fig. 3. The dashed-dotted line in (b) is the correlation estimated from filtered trajectories using a Gaussian kernel $K=A_{w} \exp \left(-t^{2} / 2 w^{2}\right)$, where $w=12$ points and $A_{w}$ is a normalization factor. 
a)

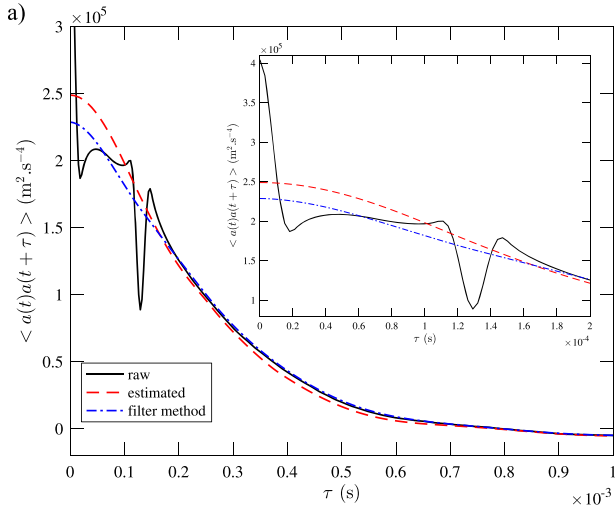

b)

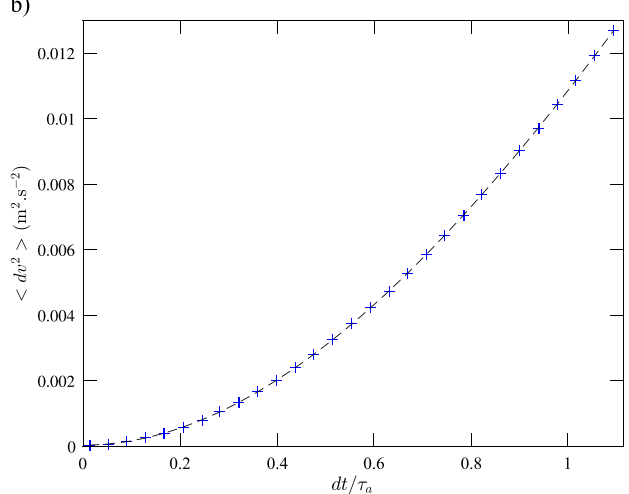

FIG. 5. (a) Acceleration auto-correlation functions estimated from the proposed method (dashed line) or directly computed by differentiating the velocity signal obtained by ELDV (continuous line). The dashed-dotted line is the correlation estimated from filtered trajectories using a Gaussian kernel $K=A_{w}$ exp $\left(-t^{2} / 2 w^{2}\right)$, where $w=20$ points and $A_{w}$ is a normalization factor. The inset is a zoom on the low values of $\tau(\mathrm{b})(+)$ : evolution of $\left\langle(d v)^{2}\right\rangle$ with $d t($ represented one point on three for clarity). The dashed line is a third order fit $\left(\alpha_{v}+\beta_{v} d t^{2}+\gamma_{v} d t^{3}\right)$. In both figures, the increments where fitted over the range $0<d t / \tau_{a}<1$.

Figure 5(a) displays the auto-correlation function of the acceleration, raw or estimated by the proposed method, for a very high Reynolds number $R e_{\lambda}=950$. The raw acceleration correlation function clearly shows the correlated nature of the noise, being strongly biased over a large range of time scales. The estimated function has been obtained by fitting over a wide range $0<d t / \tau_{a}<1$, so that a third order polynomial is required to properly fit the evolution of velocity increments with $d t$ [Fig. 5(b)]. Although such a range was chosen to be larger than the expected noise correlation time, its choice was not critical and fits over smaller ranges down to $(d t)_{\max } / \tau_{a}=0.6$ changed the values by less than 3\%. This demonstrates that if the method framework was derived assuming an uncorrelated noise, it still can be used successfully in the case of a colored noise. Indeed, the estimated function presents a shape close to that which is expected in the absence of noise and is found to be close to the auto-correlation function of filtered data from Ref. 19, which still exhibits some slight oscillations (around $\tau=0.15 \mathrm{~ms}$ ) despite the large filter width $w=20$ points. Increasing the filter width would suppress this oscillation at the cost of strongly decreasing the value at $\tau=0$, altering more the shape of the function. The rms of the acceleration estimation for new method $a^{\prime}=498.9 \mathrm{~m} \mathrm{~s}^{-2}$ is in good agreement with the value $a^{\prime}=496 \mathrm{~m} \mathrm{~s}^{-2}$ which is found in Ref. 19 by extrapolation of this quantity using different filter widths. The acceleration time scale is found to be $\tau_{a}=0.232 \mathrm{~ms}$, changing less than $0.1 \%$ when changing the fitting range from $\left[0,0.6 \tau_{a}\right]$ to $\left[0, \tau_{a}\right]$, in very good agreement with the extrapolated value $\tau_{a}=0.234 \mathrm{~ms}$ from the variable filter width method. ${ }^{19}$ Given the statistical convergence of the data, the different estimates of the time scale should be considered the same, $\tau_{a}=0.23 \mathrm{~ms}$. We would like to stress here that the choice of the fitting range was not crucial in the analysis and did not require a bias study as is the case for the extrapolated results of filtered signals.

\section{DISCUSSION AND CONCLUSION}

In this paper, we have presented a general method to estimate moments and auto-correlation functions of experimental signal derivatives rid of measurement noise. This method relies on two main assumptions as follows:

1. The signal has to be correlated on a longer time scale than the noise.

2. The sampling frequency, $f_{s}$, must be high enough so that the signal first and second order derivatives can be computed by taking increments over several $(N)$ points.

We observed in the numerical tests that this adjustable parameter $(N)$ has a small influence on the results and we estimated that $f_{s} \tau_{2}=20$ is a good choice when one is interested in the signal second derivative, $\tau_{2}$ being the correlation time of the signal second derivative to be estimated. We have tested the method in the context of Lagrangian particle tracks in turbulence, considering both first- and second-order derivatives of a time dependent signal. First, we used numerical data, artificially adding a white noise and successfully comparing quantities estimated through this method to those computed prior to noise addition. Second, tests were conducted on classical particle tracking velocimetry and extended laser Doppler velocimetry data. In the former, the noise is correlated on times much shorter than the signal, and in the latter, the noise becomes colored through a filtering operation inherent in the measurement. Whatever the case, the results are in good agreement to what is obtained by classical filtering processes, which require a long bias study specific to the data type, ${ }^{13,19}$ and we believe them to be more accurate. The method avoids subjective tuning of the filter width and choice of filter type while yielding unbiased quantities by requiring data fits in an appropriate range. While the fit range is still an adjustable parameter, we observed its impact on the results to be smaller than when filtering the data. We have shown with the numerical test that the error remains very small even while varying the fit range by a factor 7 . Another advantage of the method is an easy access to the noise magnitude. While building a new experimental setup, one can gather just enough statistics to converge second order moments to estimate the noise magnitude and try and improve the setup iteratively. With more statistics, having access to the noise correlation 
function ensures that the noise is not correlated (in cases where it is not expected to be so). We have also tested the method in a more general context of a random position signal whose firstand second-derivative auto-correlation functions are known (not shown here). The signal was generated using the 2-time Sawford model ${ }^{20}$ which solves a coupled set of Langevin equations for both the velocity and acceleration. Once again, we found an almost perfect agreement between the analytical function and the one obtained by the proposed method. The method presents some drawbacks such as added computation time. The major drawback is the need to sample the signal at a larger rate than would usually be necessary. Indeed, this offers a larger range over which $d^{2} x$ still can be related to a second-order derivative. While this is easily achieved with ELDV measurements, as the sample rate is already very high and the data are not voluminous, this can cause a limitation on the trajectories duration for typical PTV setup or other measurements based on embarked-memory cameras, making the trade-off between temporal resolution and duration even harder.

\section{ACKNOWLEDGMENTS}

This work is supported by French Research Program Nos. ANR-12-BS09-0011 and ANR-13-BS09-0009 and project Emergent PALSE/2013/26. Contribution from the European project EuHIT-European High-performance Infrastructures in Turbulence-is also acknowledged. We thank M. Bourgoin and P. Borgnat for fruitful discussions and E. Calzavarini for access to the numerical data.

${ }^{1}$ R. Chapman, "Instrumentation for analog differentiator at low frequencies," Rev. Sci. Instrum. 40(1), 95-98 (1969).

${ }^{2}$ S. Smith, Digital Signal Processing: A Practical Guide for Engineers and Scientists (Newnes, 2013).

${ }^{3}$ O. Chanal, B. Chabaud, B. Castaing, and B. Hébral, "Intermittency in a turbulent low temperature gaseous helium jet," Eur. Phys. J. B 17(2), 309317 (2000).
${ }^{4}$ G. P. Romano, R. Antonia, and T. Zhou, "Evaluation of LDA temporal and spatial velocity structure functions in a low Reynolds number turbulent channel flow," Exp. Fluids 27(4), 368-377 (1999).

${ }^{5}$ R. J. Adrian, "Particle-imaging techniques for experimental fluidmechanics," Annu. Rev. Fluid Mech. 23, 261-304 (1991).

${ }^{6}$ Springer Handbook of Experimental Fluid Dynamics, edited by C. Tropea, A. Yarin, and J. F. Foss (Springer-Verlag, Berlin, Heidelberg, 2007).

${ }^{7}$ R. Volk, N. Mordant, G. Verhille, and J.-F. Pinton, "Laser Doppler measurement of inertial particle and bubble accelerations in turbulence," Europhys. Lett. 81, 34002 (2008).

${ }^{8} \mathrm{R}$. Tsai, "A versatile camera calibration technique for high accuracy $3 \mathrm{D}$ machine vision metrology using off-the-shelf TV cameras and lenses," IEEE J. Rob. Autom. 3, 323 (1987).

${ }^{9}$ N. T. Ouellette, H. Xu, and E. Bodenschatz, "A quantitative study of threedimensional Lagrangian particle tracking algorithms," Exp. Fluids 40(2), 301-313 (2006)

${ }^{10} \mathrm{H}$. Xu, "Tracking Lagrangian trajectories in position-velocity space," Meas. Sci. Technol. 19(7), 075105 (2008).

${ }^{11} \mathrm{~N}$. Mordant, J.-F. Pinton, and O. Michel, "Time-resolved tracking of a sound scatterer in a complex flow: Nonstationary signal analysis and applications," J. Acoust. Soc. Am. 112(1), 108-118 (2002).

${ }^{12} \mathrm{~N}$. Ouellette, "Probing the statistical structure of turbulence with measurements of tracer particle tracks," Ph.D. thesis, Cornell University, 2006.

${ }^{13}$ G. A. Voth, A. La Porta, A. M. Crawford, J. Alexander, and E. Bodenschatz, "Measurement of particle accelerations in fully developed turbulence," J. Fluid Mech. 469, 121-160 (2002).

${ }^{14}$ E. Calzavarini, R. Volk, M. Bourgoin, E. Leveque, J. F. Pinton, and F. Toschi, "Acceleration statistics of finite-sized particles in turbulent flow: The role of Faxén forces," J. Fluid Mech. 630, 179-189 (2009).

${ }^{15}$ E. Calzavarini, M. Cencini, D. Lohse, and F. Toschi, "Quantifying turbulence-induced segregation of inertial particles," Phys. Rev. Lett. 101(8), 084504 (2008).

${ }^{16}$ E. Calzavarini, M. Kerscher, D. Lohse, and F. Toschi, "Dimensionality and morphology of particle and bubble clusters in turbulent flow,"J. Fluid Mech. 607, 13-24 (2008).

${ }^{17} \mathrm{~N}$. Machicoane and R. Volk, "Lagrangian velocity and acceleration correlations of large inertial particles in a closed turbulent flow," Phys. Fluids 28(3), 035113 (2016).

${ }^{18}$ N. Machicoane, R. Zimmermann, L. Fiabane, M. Bourgoin, J.-F. Pinton, and R. Volk, "Large sphere motion in a turbulent swirling flow," New J. Phys. 16, 013053 (2014).

${ }^{19}$ R. Volk, E. Calzavarini, E. Leveque, and J.-F. Pinton, "Dynamics of inertial range particles in a turbulent flow," J. Fluid Mech. 668, 223-235 (2011).

${ }^{20}$ B. L. Sawford, "Reynolds number effects in Lagrangian stochastic models of turbulent dispersion," Phys. Fluids A 3(6), 1577-1586 (1991). 